\title{
SPWM Smoothing Control Method Based on Wind Power Grid Connection
}

\author{
Zixu Fan ${ }^{1}$, Xingjun Tian ${ }^{1}$, Tong Zhao ${ }^{1}$, Zihang $\operatorname{Tan}^{1}$ and Chaozheng $\mathrm{Xu}^{1, *}$ \\ ${ }^{1}$ School of Electrical and Electronic Engineering, Shijiazhuang Tiedao University, Shijiazhuang, Hebei, 050043, China
}

\begin{abstract}
Energy is the foundation of economic development and technological development. Facing the present situation of non-renewable energy decline, wind power generation has been developed rapidly. However, the problem of unstable output voltage of wind power generation due to unstable wind speed needs to be solved, and traditional solutions cannot make the generated electric energy meet the national standards for grid-connected and off-grid operation. In this paper, the electric energy generated by wind turbine is rectified by bridge rectifier circuit and using large capacity capacitor filtering to generate DC with flat waveform. Then, using SPWM inversion technology, the normal rotation wave with the same frequency as the power grid is used as the modulation wave and according to the required voltage amplitude, and the triangle wave with appropriate duty ratio is calculated by SPWM smoothing control theory as carrier wave. Subsequently, accurate filtering is carried out and grid-connected and off-grid operation is carried out through the self-aligning device. Finally, with the help of MATLAB simulation platform, the wind turbine is simulated to work under different wind conditions, and whether the generated electric energy meets the grid-connected and off-grid operation standards is judged, which further determines the reliability and authenticity of the theory.
\end{abstract}

\section{Introduction}

Today's world energy resources are mainly coal, oil and natural gas. However, with the rapid development of society, these resources will be exhausted and can no longer meet the long-term development requirements of mankind. Various new energy sources have emerged, including wind energy, solar energy and hydropower generation. Among various forms of new energy, wind power generation has developed into a new energy industry with the most commercial development prospect at present, and has been highly valued by governments and major companies all over the world, which is conducive to becoming the key research and development direction of new energy development [1].

However, due to the uncertainty and uncontrollability of wind energy itself, the generated power quality will be seriously affected, which makes the power fail to meet the national standards for grid-connected and off-grid operation, resulting in large-scale wind abandonment and power abandonment, and the technical problems of wind power grid-connected need to be solved. Although predecessors have made great progress in the research of stabilizing the running speed of wind turbines, they invented synchronous wind turbines and doubly-fed wind turbines, which can weaken the change of $\mathrm{AC}$ frequency and amplitude from the source, but still can not eliminate the fluctuation of power quality caused by the randomness of wind energy [2]. Therefore, it is particularly important to treat the generated electric energy to meet the national standards.

The total installed capacity of wind turbines in China in recent years is shown in Figure 1.

New installed capacity of global wind turbines from 2001 to 2020 (unit MW)

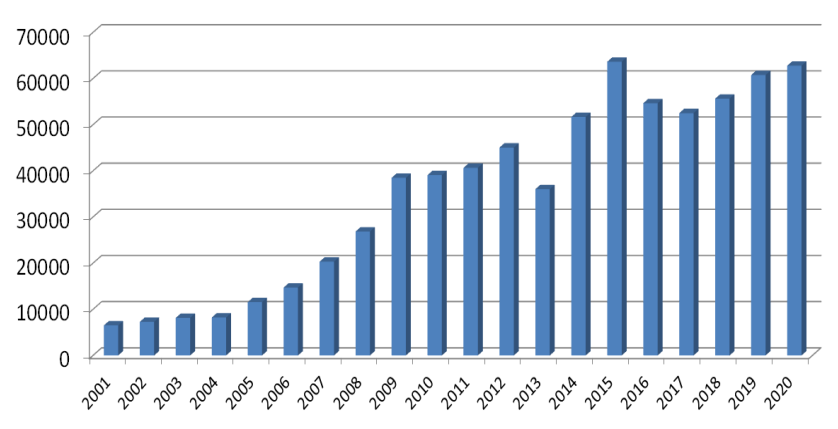

Fig. 1. Statistical Chart of Installed Wind Turbines in China

\section{Materials and Methods}

\subsection{General Ideas and Methods}

Due to the uncertainty of wind speed and direction, the electricity generated by wind turbine is alternating current with changing frequency, which is difficult to be improved by simple filter device. The device developed in this paper can effectively control the frequency, amplitude and harmonic components of output power, so

\footnotetext{
${ }^{*}$ Corresponding author: std_ele@163.com
} 
as to achieve the standard of grid-connected or off-grid operation and make the power output effective. The general idea is shown in Figure 2.

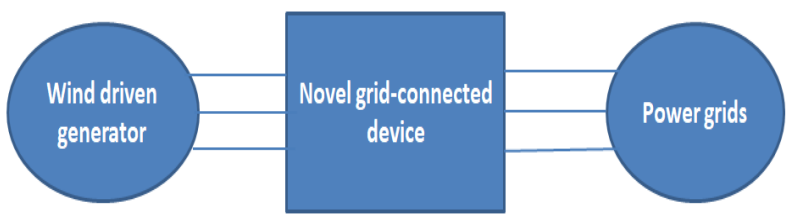

Fig.2. General train of thought

The grid-connected system consists of bridge diode rectifier circuit, SPWM inverter circuit, second-order low-pass filter circuit, IGBT trigger circuit and so on [3]. Two AC power supplies with different voltage amplitude and different frequencies are connected in series to simulate the fan port voltage under random wind conditions. The model diagram is shown in Figure 3.

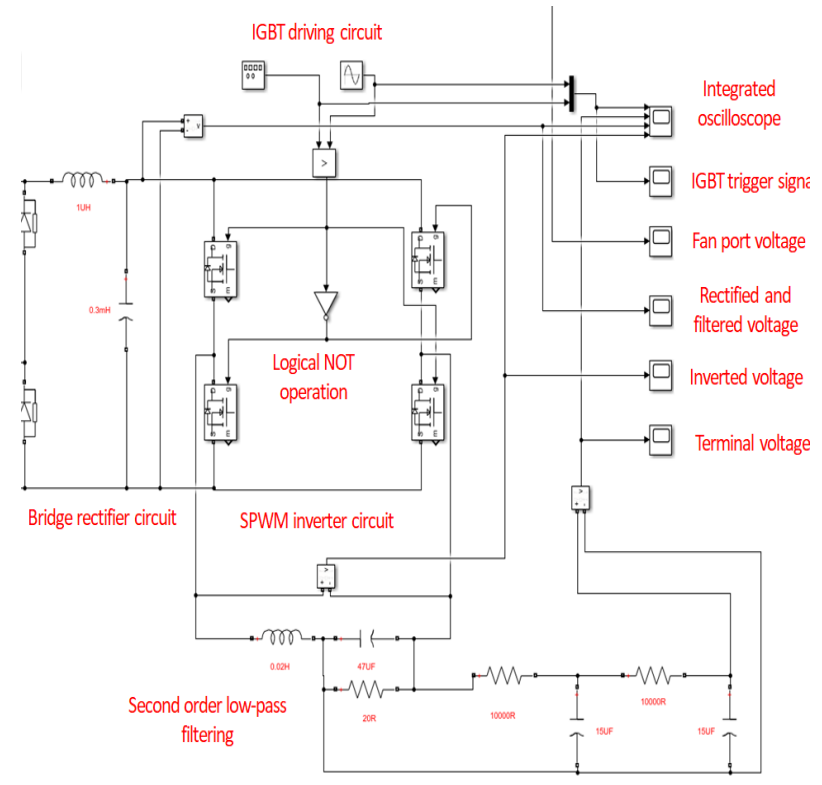

Fig.3. Simulation model diagram

\subsection{Terminal Voltage Control Mode}

At present, thyristor inverter circuit is mostly used in the inverter mode, but the disadvantage of this topology is that the power quality of the power grid is reduced, and this structure needs to add additional devices in reactive power compensation and harmonic suppression. SPWM inverter based on IGBT is a fully controlled inverter, which can realize self-commutation, has little influence on power quality, can improve power quality and has high power factor, and is more suitable for high-power environment in power system. Therefore, SPWM inverter technology is adopted in this paper.

Monopolar control theory mostly adopts modulation method, which is difficult to form a stable control system and relatively complex, which is not conducive to accurate control of terminal voltage. Therefore, this paper adopts bipolar control, which can smoothly control the terminal voltage through theoretical derivation and calculation.
At present, there are two ways of SPWM control, namely unipolar control and bipolar control [4]. The two control modes are shown in Figure 4 and Figure 5.

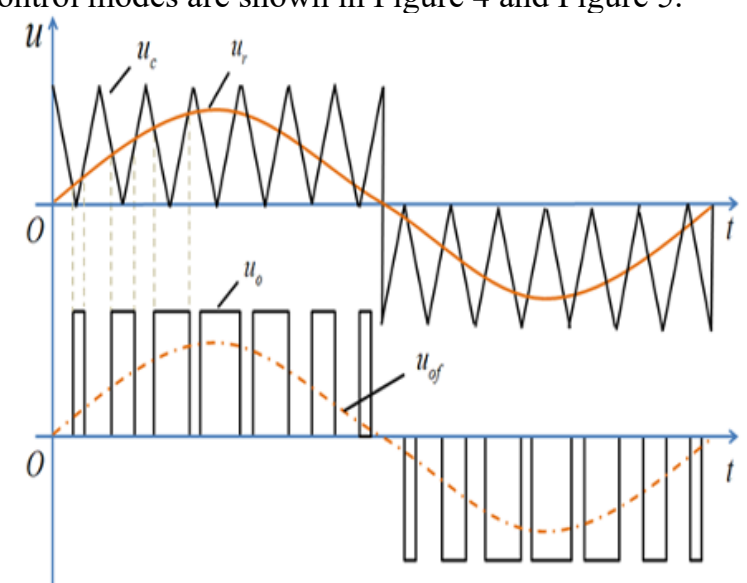

Fig.4. Schematic diagram of unipolar control

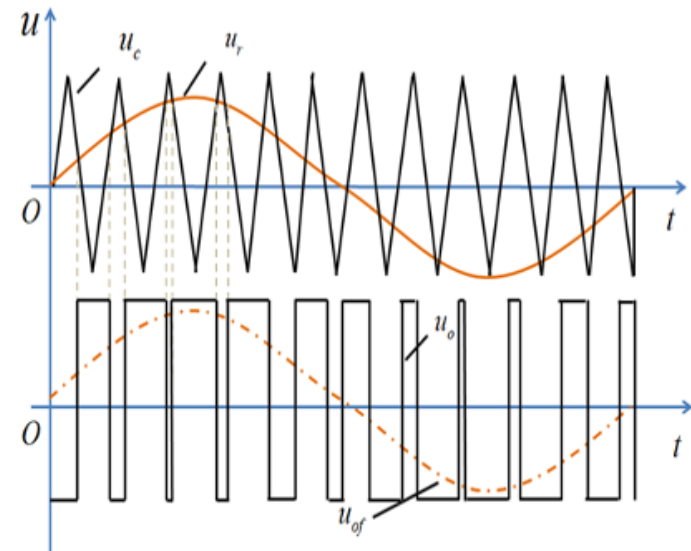

Fig.5. Schematic diagram of bipolar control

\subsection{Terminal voltage control theory}

The amplitude and frequency of the voltage obtained after inversion are determined by SPWM modulation method. The voltage amplitude is determined by carrier ratioand [5]. The voltage frequency is controlled by modulation wave. Therefore, the correlation calculation between modulated wave and carrier wave is particularly important for smoothing the amplitude and frequency of control voltage [6].

The principle of SPWM regular sampling control method is shown in Figure 6.

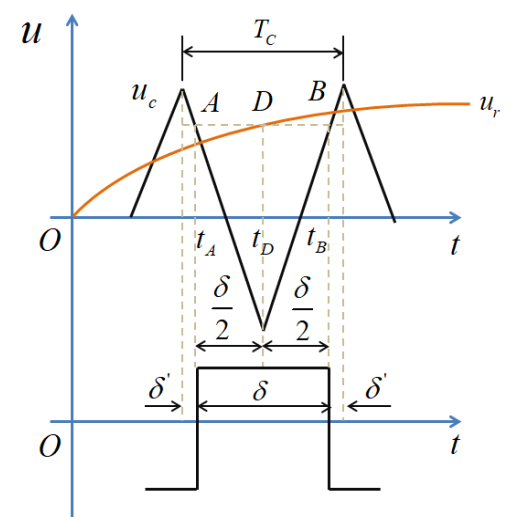

Fig.6. Schematic diagram of SPWM regular sampling method 
Take a sampling period between two peaks of triangular wave, corresponding pulse width is $\delta$. Let the sine wave of the control signal of the kth cycle be.

$$
u=A \sin \left(100 \pi \cdot t_{\mathrm{D}}\right)
$$

Let the peak value of the carrier be $\mathrm{B}$, and regard the triangular wave as a periodic piecewise function, in which the midpoint of the pulse is $K T_{z}$.

$$
A \sin \left(100 \pi \cdot K T_{\mathrm{Z}}\right)=\frac{2 B}{T_{\mathrm{Z}}} t-(k-1) B
$$

Expand SPWM pulse sequence into Fourier series.

$$
\begin{gathered}
u(t)=\sum_{k=1}^{\infty} U_{k m} \sin \left(k \omega_{1} t\right)(k=1,3,5 \ldots \ldots) \\
U_{k m}=\frac{2}{\pi} \int_{0}^{\pi} u(t) \sin k w_{1} d w_{1} t
\end{gathered}
$$

For $n$ pulses of unipolar SPWM waveform, In which the phase of the center point of the $i$ th pulse is $\theta_{i}=(2 i-1) \pi / 2 n$, it can be obtained that the coordinates of the starting points of the $i$ th pulse are $\theta_{i}-1 / 2 \delta_{i}=(2 i-1) \pi / 2 n-1 / 2 \delta_{i}$ the endpoint coordinates are $\theta_{i}+1 / 2 \delta_{i}=(2 i-1) \pi / 2 n+1 / 2 \delta_{i}$.

Take them as the upper and lower limits of the integral function and substitute them into the $U_{k m}$ formula.

$$
U_{k m}=\frac{2}{\pi} \sum_{i=1}^{n} \int_{\theta_{i}-\frac{1}{2} \delta_{i}}^{\theta_{i}+\frac{1}{2} \delta_{i}} \sin \left(k \omega_{1} t\right) \cdot \mathrm{d} \omega_{1} t
$$

Finally get.

$$
u(t)=\sum_{k=1}^{\infty} \frac{2 U_{S}}{k \pi} \sum_{i=1}^{n}\left[\sin \frac{(2 i-1) k \pi}{2 n} \cdot \sin \frac{k \delta_{i}}{2}\right] \cdot \sin \left(k \omega_{1} t\right)(6)
$$

According to this formula, the direct relationship between the amplitude and of the output fundamental voltage can be obtained, so the amplitude of modulation amplitude can be calculated and adjusted to change each pulse width, thus smoothly adjusting the amplitude of the output fundamental voltage of inverter.

\section{Results \& Discussion}

According to the required voltage, the modulated wave parameters are calculated and brought into the Matlab simulation model [7]. So that the carrier wave and modulated wave waveform can be obtained, and then the SPWM control waveform can be obtained, as shown in Figure 7 and Figure 8.

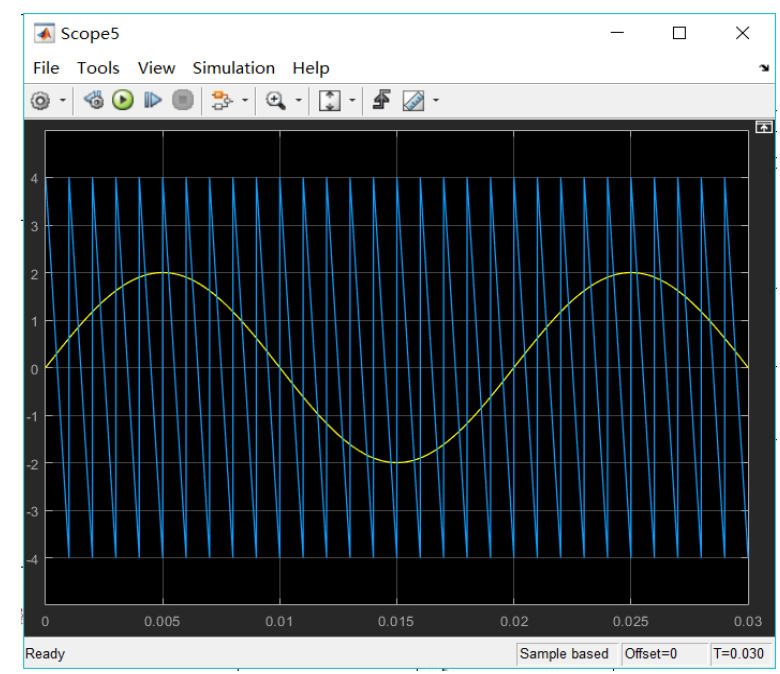

Fig.7. Carrier and modulation waveform diagram

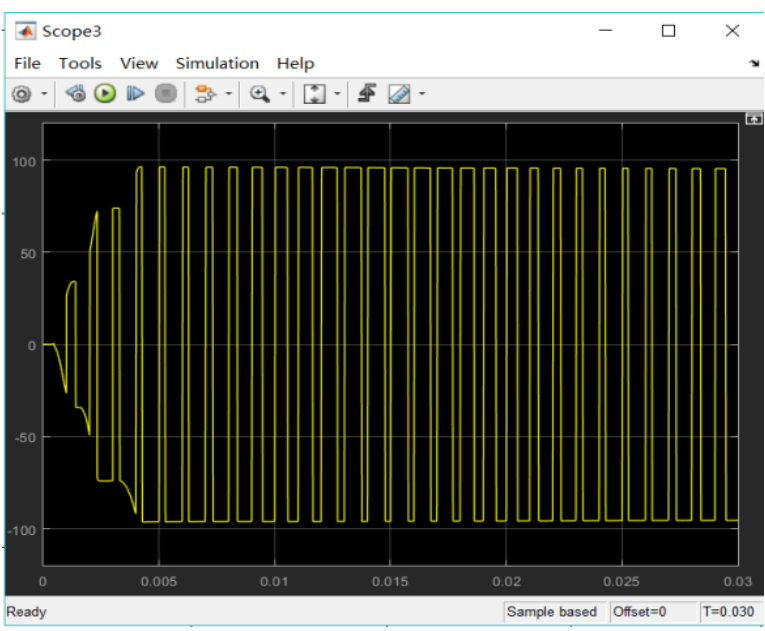

Fig.8. SPWM waveform diagram

Through the simulation model, a series of treatments on the power of the fan port can obtain stable sinusoidal alternating current, and the voltage waveforms before and after treatment can form obvious contrast. The voltage amplitude and frequency before treatment change with the rotating speed of the wind turbine, which is extremely unstable, while the voltage amplitude and frequency after treatment are extremely stable, and the power quality meets the national standard. The specific figures are shown in Figure 8 and Figure 9.

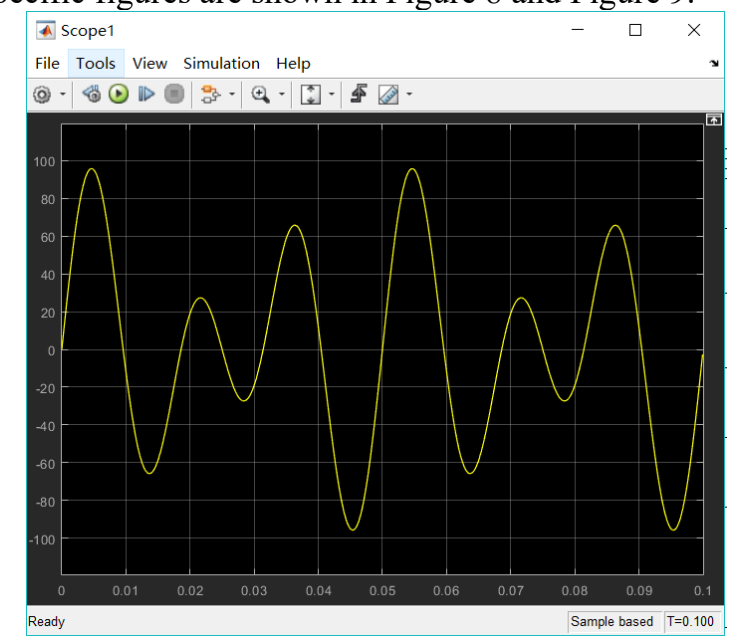

Fig.9. Voltage waveform diagram of fan port 


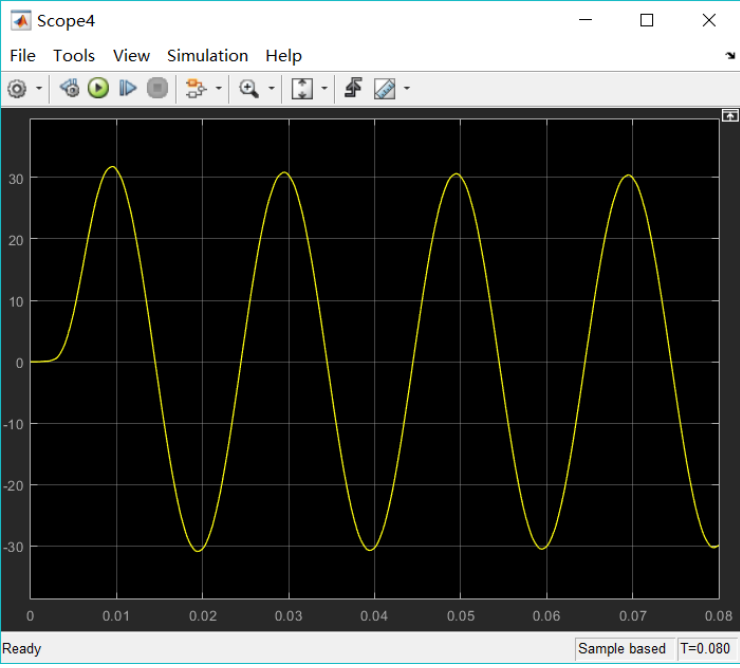

Fig.10. Voltage waveform diagram after processing

Through FFT function, Fourier analysis is performed on the processed output voltage, and the result is shown in Figure 11.

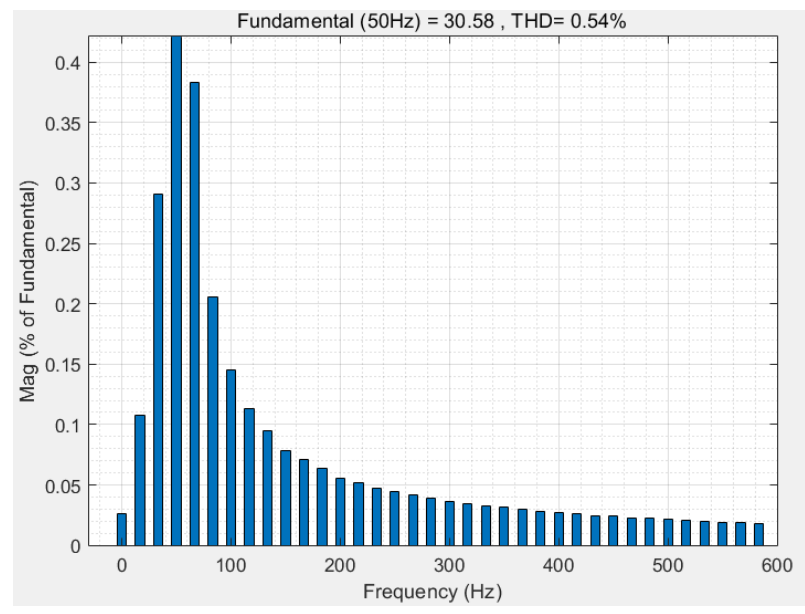

Fig.11. FFT analysis result chart

It can be seen that the obtained voltage waveform component is mainly $50 \mathrm{~Hz}$ sinusoidal alternating current, and it can be seen that the distortion rate THD is $0.54 \%$, which meets the grid-connected and off-grid operation standards stipulated by the State Grid.

\section{Conclusions}

In order to effectively control the frequency, amplitude and harmonic components of the output power of wind power generation, and achieve the standard of gridconnected or off-grid operation, this paper deduces the SPWM control theory and builds a simulation model. Finally, through FFT analysis of the output waveform, the distortion rate of the output voltage waveform is obtained, which is highly in line with the national power grid-connected and off-grid operation standards, and has great practical significance. It can greatly reduce the abandoned air volume, greatly increase the power generation, and make the new energy more fully utilized.

\section{References}

1. Wang, C. (2020) Wind power generator and wind power generation control technology. Hubei Agricultural Mechanization, 18: 33-34. Podani, J. (1994) Multivariate Data Analysis in Ecology and Systematics. SPB Publishing, The Hague.

2. Lin, T. (2021) Discussion on grid-connected technology of wind power generation and key points of power quality control. Industrial \& Science Tribune, 20: 33-34.

3. Zhao, S. (2015) Modeling and Simulation of SPWM Inverter System on Rotor Side of Fan. Communication Power Supply Technology, 41: 8992.

4. Zhou, Y. (2016) Power Electronic Technology. Machinery Industry Press, The Beijing.

5. Lin, W. (2005) Advanced Power Electronics. Machinery Industry Press, The Beijing.

6. [6] Jiang, N. (2020) Research on Influencing Factors of Output Common Mode Voltage of SPWM Inverter. Electric Power Big Data, 23: 74-84.

7. Lei, H. (2021) Design of Simulation Wind Power Generation Control System. Practical Electronics, 7: 13-16. 